\title{
In-Hospital Outcome of Older and Younger Patients with Acute Coronary Syndrome
}

\author{
SM EftarJahan Kabir ${ }^{1}$,Abdul Wadud Chowdhury² Md.Gaffar Amin'2,Mohammad Sarwar \\ Alam $^{1}$, Khandker Md.Nurus Sabah ${ }^{2}$, Abu Sadique Abdullah ${ }^{3}$, Mofazzal \\ Hossain $^{4}$, AbuZahid $^{5}$, Chowdhury Omar Faruk $^{6}$, Tunaggina Afrin Khan ${ }^{7}$ \\ ${ }^{1}$ Department of cardiology, National Institute of Cardiovascular Diseases, ${ }^{2}$ Department of Cardiology, \\ Dhaka Medical College, Dhaka, ${ }^{3}$ Department of cardiology, Bangabandhu Sheikh Mujib Medical \\ University, Dhaka, ${ }^{4}$ Upozilla Health Complex,Madan, Netrokona, ${ }^{5}$ Upozilla health Complex \\ Gangachara, Rangpur, ${ }^{6}$ Emergency Department, MAGOsmani Medical College, Sylhet, ${ }^{7}$ Anowar Khan \\ Modern Medical College and Hospital
}

Key words: Age, Acute coronary syndrome, Ischaemic heart disease.

\begin{abstract}
:
Background: Acute coronary syndrome is a cardiac emergency. It is increasingly common in younger peoples. Management of elderly peoples is difficult due to their associated comorbidity. This study tried to compare the in-hospital outcome of the younger and older patients with acute coronary syndrome.

Methods: The study was a comparative cross sectional study. Clinical and biochemical evaluation was done in hospital settings. A total number of 120 patients were included in the study and divided into two groups according to distribution of age. In group I there were elderly groups of aged $>60$ yrs. and in group II there were patients within the age 40 to 60 yrs. All the data were collected systematically in a preformed data collection form.

Results: Group I populations had more in hospital stay and more complications than group II.

Conclusion: The study revealed significant association with age and outcome of Acute coronary syndrome patients. Complications of acute coronary syndrome increase as the age of the patients increases.
\end{abstract}

(Cardiovasc. j. 2015; 8(1): 49-52)

\section{Introduction:}

Even in the developed countries of the world, Ischaemic heart disease (IHD) is the leading cause of death among patients aged $>65 \mathrm{yrs}^{1}{ }^{1}$ In future there will be a dramatic surge of patients with IHD due to ageing of population. ${ }^{2}$ Because of the continuing increase in life expectancy, many of these patients will be aged $>75$ yrs. Elderly peoples comprise only $9 \%$ of all clinical trials and only about $50 \%$ of trials enroll patients above the age of $75 .{ }^{3}$ Data regarding coronary reperfusion in older acute coronary syndrome (ACS) patients are limited and comprise mostly of subset of analysis from major trials and retrospective studies. ${ }^{4}$ Thus information is sparse to guide the care of this high risk ACS patients. Moreover elderly peoples present in atypical way so that initial diagnosis of ACS is delayed. Medication side effects are common in elderly and complication in relation to treatment is also high. So outcome depends upon the diseases and upon the treatment itself. ACC/ AHA emphasizes intensive, early medical and interventional therapy for the high risk group. The elderly groups are known to be at high risk for short term events, but community practice patterns do not follow this frequently. For gaining of quality of life it is important to prompt diagnoses and treatment of the patients with ACS. Therefore there should be comprehensive knowledge and available evidence and guideline for treatment of elderly population.

Methods:

This cross sectional observational study was conducted at department of cardiology in Dhaka Medical College Hospital from April 2011 to March 2012 with the objective to assess in- hospital

Address of Correspondence: Dr. SM EftarJahan Kabir, Department of Cardiology, National Institute of Cardiovascular Diseases, Dhaka, Bangladesh. Email: eftarjahan@gmail.com 
outcome of older and younger patients with ACS. Study population was all the patients with ACS in coronary care unit, Department of cardiology Dhaka Medical College Hospital within the study period. Patients aged within 40 years to 60 years, having previous history of MI, undergone Percutaneous coronary intervention or pharmacological thrombolysis, coronary artery bypass graft surgery, cardiomyopathy and other comorbidity were excluded. After fulfilling all the inclusion and exclusion criteria index patients were included. Patients aged $>60$ yrs. were taken in group-I and patients $<40$ yrs. were taken in groupII. Sample size was 120. Patients were evaluated both clinically and by investigations. Hospital stay time, heart failure, arrhythmia, conduction defect, cardiogenic shock and death were evaluated as outcome variables.

Data was collected properly and systematically analyzed by SPSS version 12 . Test statistics used to analyze the data were descriptive statistics, chi square and unpaired t-test. Level of significance was set at .05.

\section{Results:}

There were no statistically significant differences between the groups regarding sex, clinical diagnoses, family history, electrocardiogram, biochemical findings (Troponin-I, serum creatinine, fasting lipid profile).

Distribution of patients was same. Regarding symptoms chest pain was present in all patients; dyspnoea was more in older ones. Other symptoms were similar in both groups $(\mathrm{p}>0.05)$. Regarding pulse rate there was no significant difference between two groups but the systolic and diastolic pressure of the patients in group-I were significantly higher than group-II ( $\mathrm{p}=.025$ for systolic blood pressure and $\mathrm{p}=0.019$ for diastolic blood pressure).Number of smoker had significantly higher in group II ( $\mathrm{p}=0.010)$; on the contrary, groupI had significantly higher number of study subjects with hypertension $(p=0.024)$, diabetes mellitus $(p<0.001)$, dyslipidaemia $(p<0.001)$ than group-II. Family history of premature coronary artery disease did not differ significantly between the two groups $(\mathrm{p}=0.224)$. Mean random blood sugar of

Table-I

Clinical diagnosis of the study population $(n=120)$.

\begin{tabular}{lcccc}
\hline Clinical diagnoses & Group-I(n=60)N $(\%)$ & Group-II $(\mathrm{n}=60) \mathrm{N}(\%)$ & Total $(\mathrm{n}=120) \mathrm{N}(\%)$ & $\mathrm{p}$ - value \\
\hline NSTEMI & $12(20.0 \%)$ & $13(21.7 \%)$ & $25(20.8 \%)$ & 0.822 \\
STEMI & $36(60.0 \%)$ & $33(55.0 \%)$ & $69(57.5 \%)$ & 0.580 \\
Unstable angina & $12(20.0 \%)$ & $14(23.3 \%)$ & $26(21.7 \%)$ & 0.658 \\
\hline
\end{tabular}

NSTEMI- Non ST elevation myocardial infarction. STEMI- ST elevation myocardial infarction.

Table-II

In-hospital outcomes of the study population $(n=120)$.

\begin{tabular}{lcccc}
\hline In-hospital outcome & Group-I(n=60)N $(\%)$ & Group-II $(\mathrm{n}=60) \mathrm{N}(\%)$ & Total(n=120)N $(\%)$ & $\mathrm{p}$ - value \\
\hline Heart failure & $19(31.7 \%)$ & $9(15 \%)$ & $28(23.3 \%)$ & 0.031 \\
Arrhythmia & $9(15.0 \%)$ & $7(11.7 \%)$ & $16(13.3 \%)$ & 0.591 \\
Cardiogenic shock & $8(13.3 \%)$ & $2(3.3 \%)$ & $10(8.3 \%)$ & 0.048 \\
Conduction defect & $10(16.7 \%)$ & $3(5.0 \%)$ & $13(10.8)$ & 0.040 \\
Death & $5(8.3 \%)$ & $3(5.0 \%)$ & $8(6.70 \%)$ & 0.061 \\
\hline
\end{tabular}

Table-III

Comparison of hospital stay of the study population $(n=120)$.

\begin{tabular}{lccc}
\hline Duration of hospital stay & Group-I $(\mathrm{n}=60)$ & Group-II $(\mathrm{n}=60)$ & $\mathrm{p}$ - value \\
\hline Mean $\pm \mathrm{SD}$ & $7.35 \pm 1.55$ & $6.46 \pm 2.09$ & 0.010 \\
\hline
\end{tabular}


group I was significantly higher than group II $(p=0.017)$. The mean serum creatinine level of patients between the two groups showed no significant differences ( $p=0.244)$. Serum level of high density lipoprotein (HDL)-cholesterol was significantly lower in group I than group2 $(\mathrm{p}<0.001)$. Difference in ejection fraction between the two groups was not statistically significant $(\mathrm{p}=0.0972)$.

The study subjects of group I developed significantly more number of heart failure, cardiogenic shock and conduction defect than group II; however no significant differences was observed in case of arrhythmia $(\mathrm{p}=0.591)$ and death $(\mathrm{p}=0.061)$. In comparison of duration of hospital stay between two groups: the study subjects of group I had to stay at hospital more days than group II $(\mathrm{p}=0.010)$.

Among in-hospital outcomes of patients with ACS, age of the patients attributes $74.1 \%$ in the development of heart failure, $85.5 \%$ in the development of cardiogenic shock, $79 \%$ in the development of arrhythmia, $83.8 \%$ in the development of conduction defect and $82.6 \%$ in death. Smoking attributes in the development of heart failure, cardiogenic shock, arrhythmia, conduction defect and death respectively (69.1\%,80.1\%,77\%,76.4\%).Hypertension attributes in development of heart failure, cardiogenic shock, arrhythmia, conduction defect and death $(68.7 \%, 84,2 \%, 80.3 \%, 81.8 \%, 81.2 \%)$ respectively. Family history of CAD attributes heart failure, cardiogenic shock, arrhythmia, conduction defect and death respectively $(69.1 \%, 86.8 \%, 82.4 \%$, $81.0 \%, 80 \%)$. Dyslipidaemia attributes in the development of heart failure, cardiogenic shock, arrhythmia, conduction defect and death respectively $(76.1 \%, 82.5 \%, 77.6 \%, 82.5 \%, 82.4 \%$, $77.7 \%$ ). Diabetes mellitus attributes in the development of heart failure, cardiogenic shock, arrhythmia, conduction defect and death respectively $(65.7 \%, 90.1 \%, 81 \%, 85.1 \%, 82.9 \%)$.

\section{Discussion:}

The study was intended to compare the various clinical presentations, in-hospital outcomes and its influencing factors between older and younger patients with ACS. The male participants were more than female in both the groups. This observation reflects the usual fact that ACS is more prevalent in male than female. ${ }^{5}$
Among the presenting complaints chest pain was present in all patients in both groups. More patients of the younger group presented with only chest pain and the older group presented with only chest pain and dyspnea. Sozia et al and Schoenenberger et al found similar findings in their study. Sozia et al conducted a prospective observational cohort study with 869 patients presenting with suspected ACS over a six month period. Older patients less likely to present with chest pain and more likely present with breathlessness or collapse. ${ }^{6,7}$

Group II had significantly higher number of study subjects with smoking habit $(\mathrm{p}=0.010)$ than groupI. Conversely group I had significantly higher number of study subjects with hypertension $(p=0.024)$, diabetes mellitus $(p=0.001)$ and dyslipidaemia $(p<0.001)$ than group II. However family history of IHD did not differ significantly between groups $(p=.0224)$. Panduranga et al analyzed data from 1579 patients with ACS. Our findings were consistent with that of them. ${ }^{8}$ The difference in serum troponin- I level was not statistically significant $(9.32 \pm 7.83$ and $10.94 \pm 7.46, p=0.247)$. The mean random blood sugar level of group I was significantly higher than group II (7.88 \pm 2.77 vs $6.92 \pm 1.29 \mathrm{mmol} / \mathrm{l}, \mathrm{p}=0.017)$, but serum creatinine level was not statistically significant $(p=0.244)$. Our observations are in the line of Panduranga et al. Older patients present with higher frequencies of diabetes and renal impairment than younger patients. ${ }^{8}$ There were no significant differences between group I and group II in the concentration of total cholesterol,low density lipoprotein cholesterol and triglyceride. However serum level of HDL cholesterol was significantly lower in group I than in group II $(\mathrm{p}=0.001)$.Our findings are partly supported by Panduranga et al. They analyzed data of 1579 consecutive patients with ACS and observed that older patients had higher frequencies of hyperlipidemia $(\mathrm{p}<0.001)$, than younger counterpart. However Tungsubutra et al did not support the findings of present study. The mean \pm duration SD of hospital stay of patients of Group I and Group II were $7.35 \pm 1.55$ and $6.46 \pm 2.09$ respectively. Group I subjects had to stay in hospital more days than group II $(\mathrm{p}=0.010)$.We considered heart failure, arrhythmia, cardiogenic shock, conduction defect, death, duration of hospital stay and discharge as inhospital outcome. The study subjects of group I 
developed significantly more number of heart failure $(31.7 \%$ vs $15 \%, \mathrm{p}=0.031)$, cardiogenic shock $(13.3 \%$ $3.3 \%, \mathrm{p}=0.048)$ and conduction defect $(16.7 \% v s 5.0 \%, \mathrm{p}=0.040)$ than group II. However no significant differences was observed in case of arrhythmia (15.0\%vs $11.7 \%, \mathrm{p}=0.059)$ and death ( $8.3 \%$ vs $5.0 \%, p=0.061)$ between two groups. Our study was supported by Sozia et al, Al-Murayeh et al, Schoenenberger et al, Halon et al, Skolnick and Panduranga et al. Sozia et al in their prospective observational study found that older patients with suspected ACS were more likely to have worse outcomes than their younger counterpart despite having fewer major risk factors. ${ }^{6}$

Al-Murayeh et al retrospectively reviewed database of 924 Saudi patients with ACS to investigate shortterm outcome of ACS in young population. The results revealed that in hospital one young patient had acute ischaemic stroke and one elderly patient died, $22.4 \%$ of the study subjects and $32 \%$ of control group were discharged with clinical diagnoses of heart failure. Schoenenberger et al found that only three younger patients (1.5\%) died during hospitalization which was significantly less than in older age group. Major adverse cardiac events occurred in only $2.1 \%$ of young patients and $9.0 \%$ (four fold increase) in older age group $(p=0.001)$. Very elderly group had more often heart failure (33.3\%vs19.4\%) and renal dysfunction (21.6\% vs $12.3 \%$ ) than comparatively less elderly group. ${ }^{9}$ The very elderly patients were sicker on admission and had poor outcome. ${ }^{9}$ Skolnick et al studied 5,557 patients with NSTEMI age 90 yrs and compare in hospital outcome with a patients aged 75 to 89 yrs. Although both groups had much in common, in comparison with the younger elderly, the older elderly was less likely to be diabetic, smokers or obese. The older elderly were more likely die (12.0\% vs $7.8 \%)$ and experience more frequently adverse events (26.8\% vs $21.3 \%)$ during hospitalization. ${ }^{10}$ ACS among young adult is relatively low when compare with elderly population. ${ }^{8}$ Heart failure and atrial fibrillation were common complication during hospital stay and were significantly more common with higher age, whereas recurrent ischaemia and reinfarction displayed only weak or non-existent associations with age. ${ }^{11}$

\section{Study limitation:}

Sample size was small. Study was done in a tertiary hospital which does not represent the general population of the whole country. The study was conducted in a single center. There was a skip of population group aged between $40-60 \mathrm{yrs}$. so the presenting symptoms, risk factors and in-hospital outcomes of that age group cannot be predicted from the study.

\section{Conclusion:}

The comparative cross-sectional study shows that older ACS patients have poor in-hospital outcomes than younger ones. To reduce the fatal outcome proper knowledge and facilities are essential. Age specific treatment protocol may be produced too. This will improve morbidity and mortality results; and more study should be done including older patients.

\section{Conflict of Interest - None.}

\section{References:}

1. Kesteloot H, San S, KromhoutD. Dynamics of cardiovascular mortality in western and Eastern Europe between 1970 and 2000. Eur Heart J 2006; 27:107-113.

2. Escaned J, Ryden L, Zamorano JL, Fuster V, Gitt AK, Fernandez-Avile's F et al. European Conference on the future cardiology. Trends and contexts in European cardiology practice for next 15 yrs: the Madrid Declaration: a report from the European Conference on the future of cardiology, Madrid,2-3 June 2006. Eur Heart J 2007,28:634-637.

3. DeWood MA, Spores J, Hensley GR. Coronary arteriographic findings in acute transmural myocardial infarction. Circulation1983 Aug; 68(2 pt 2):139-249.

4. CDC. Public health and ageing: trends in aging: United states and worldwide. MMWR 2003: 52:101-106.

5. Avezum A, Makdisse M, Spencer F, Gore JM, Fox KAA, Montalesscot G. Impact of age on management and outcome of acute coronary syndrome: from the global registry of acute coronary events (GRACE). Am Heart J 2005;149:67-63.

6. Sozia RL, Lesile SJ, Harlid K, Peden NR, Hargreaves AD. Age-dependent Differences in Presentation, Risk factor profile and Outcome of suspected Acute Coronary Syndrome. Journal of American Geriatrics Society 2005;53: 1961-1965.

7. Schoenberger AW, Radovanovic D, Stauffer JC, Windecker S, Urban P, Gregor Niedermaier G et al. Acute coronary syndrome in young patients: presentation treatment and outcome. Int J Card 2011; 148: 300-304.

8. Panduranga P, Sulaiman K, Al-Zakwani I and Abderahman S. Acute Coronary Syndrome in Young Adults from Oman: Results from the Gulf Registry of Acute Coronary Events. Heart views 2010;11:93-98.

9. Halon DA, Adawi S, Dobrecky-Mery I, Lewis BS. Importance of increasing age on the Presentation and Outcome of Acute Coronary Syndrome in Elderly Patients. J Am Coll Cardiol 2004;43:346-352.

10. Rosengren A, Wallentin L, Gitt AK, Behar S, Battler A, Hasadi D et al. Sex , age and clinical presentation of acute coronary syndromes. Eur Heart J 2004;25: 663-670. 